\title{
A SHARP CONVERGENCE ESTIMATE FOR THE METHOD OF SUBSPACE CORRECTIONS FOR SINGULAR SYSTEMS OF EQUATIONS
}

\author{
YOUNG-JU LEE, JINBIAO WU, JINCHAO XU, AND LUDMIL ZIKATANOV
}

\begin{abstract}
This paper is devoted to the convergence rate estimate for the method of successive subspace corrections applied to symmetric and positive semidefinite (singular) problems. In a general Hilbert space setting, a convergence rate identity is obtained for the method of subspace corrections in terms of the subspace solvers. As an illustration, the new abstract theory is used to show uniform convergence of a multigrid method applied to the solution of the Laplace equation with pure Neumann boundary conditions.
\end{abstract}

\section{INTRODUCTION}

Many mathematical models in practice lead to symmetric and positive semidefinite problems. Simple examples include the variational formulations (or their discretizations) of boundary value problems such as the Laplace equation with pure Neumann boundary conditions (see Bochev and Lehoucq [3]) and the linear elasticity equations with pure traction boundary conditions. Other examples of singular problems are provided by the systems of equations corresponding to the generalized finite element discretizations (see [1, 20, 19, 20, 21] and [26]).

For singular systems, direct methods based on Gaussian elimination may not be appropriate and iterative methods can be much more efficient. The objective of this paper is to study the convergence of the successive subspace correction methods 25, 24 for semidefinite problems. Classic iterative methods such as the Gauss-Seidel method, and many multigrid and domain decomposition methods fall into this category of methods.

The convergence properties of the subspace correction methods for symmetric and positive definite problems have been a subject of extensive research during the last twenty years, since these iterative techniques provide very efficient solvers for many practical problems; see e.g., Hackbusch [9], Xu [25], Yserentant 28, Bramble

Received by the editor March 29, 2003 and, in revised form, July 31, 2006.

2000 Mathematics Subject Classification. Primary 65J10; Secondary 65F10.

The authors were supported in part by NSF Grant No. DMS-0209497 and Center for Computational Mathematics and Applications, Penn State University.

The first author was supported in part by National Science Foundation DMS-0609655.

The second author was supported in part by NSFC 10501001 and SRF for ROCS, SEM.

The third author was supported in part by National Science Foundation DMS-0609727 and DMS-0619587, NSFC-10528102, and Research Award for National Outstanding Youth (Class B) by National Science Foundation of China.

The fourth author was supported in part by National Science Foundation DMS-0511800. 
and Zhang [4, Trottenberg, Oosterlee and Schüller 23, Toselli and Widlund 22. and the references cited therein.

Although, the convergence theory for the subspace correction methods applied to symmetric and positive definite problems is rather complete, there are not so many results available currently for the advanced iterative methods for semidefinite problems. Convergence studies of the classical iterative methods for semidefinite problems are presented in [10, 2], 12, and 6]. An algebraic convergence analysis for the multiplicative Schwarz methods for semidefinite problems, can be found in recent works by Chang and Sun [18, Marek and Szyld [13], and Nabben and Szyld 14. Related results are also found in Nepomnyaschikh [15], where a version of the Schwartz alternating algorithm for a special class of semidefinite variational problems is considered.

The new theory in this paper has many distinctive features in comparison with the existing results on convergence of iterative methods for semidefinite problems in the literature. First of all, our analysis provides a quantitative convergence rate estimate of general iterative methods in the subspace correction framework. In particular, our convergence results can be applied to study the convergence of many iterative methods, including multigrid methods and domain decomposition methods. Second, our result is sharp as it is given as an identity for the norm of the error transfer operator under minimal assumptions on subspace solvers. These assumptions are equivalent to the energy norm convergence of the general iterative process as discussed in the recent work [11.

Our convergence analysis for the subspace correction method is an extension of the study made by $\mathrm{Xu}$ and Zikatanov [24] for the convergence rate estimate of the subspace correction methods for symmetric and positive definite problems. Such an extension, is however surprisingly not straightforward, and requires many new technical tools.

Throughout the paper, we shall use the following standard notation. Given a Hilbert space $V$ with an inner product $(\cdot, \cdot)$ and an induced norm $\|\cdot\|$, for any closed space $W \subset V, W^{\perp}$ denotes the orthogonal complement of $W$ with respect to the inner product, $(\cdot, \cdot)$; for two subspaces $N$ and $W$ of $V$ with $N$ being a closed subspace of $W, W / N$ denotes the quotient space of $W$ and $\bar{W}$ is the closure of $W$ with respect to the norm $\|\cdot\|$. For a bounded operator $T: V \mapsto V, \mathcal{N}(T)$ and $\mathcal{R}(T)$ denote the null space of $T$ and the range of $T$ respectively.

Let $\Omega \subset \mathbb{R}^{d}$ be an open and bounded domain. $L^{2}(\Omega)$ denotes the space of square integrable functions and $H^{1}(\Omega)$ denotes the standard Sobolev space consisting of square integrable functions with square integrable (weak) derivatives of first order. $(\cdot, \cdot)_{0}$ and $(\cdot, \cdot)_{1}$ denote the usual $L^{2}(\Omega)$ inner product and $H^{1}(\Omega)$ inner product, respectively. Also $\|\cdot\|_{0},\|\cdot\|_{1}$ and $|\cdot|_{1}$ denote the $L^{2}$ norm, $H^{1}$ norm and $H^{1}$ seminorm, respectively.

Following [25], we write $x_{1} \lesssim y_{1}$ and $x_{2} \gtrsim y_{2}$ whenever there exist constants $c_{1}$ and $c_{2}$, independent of important parameters such as number of subspaces and unknowns such that

$$
x_{1} \leq c_{1} y_{1} \quad \text { and } \quad x_{2} \geq c_{2} y_{2} .
$$

The rest of this paper is organized as follows. In Section 2, a variational framework is introduced for the semidefinite problems and the method of successive subspace corrections. After some technical results are presented in Section 3, the convergence theory of the proposed method will be given in Section 4 . The analysis 
of multigrid method for the Laplace equation with the pure Neumann boundary conditions is given in Section 5 Some concluding remarks are made in Section 6 A few illustrative examples, as well as an auxiliary result related to the symmetric and positive definite system of equations are provided in the appendix.

\section{VARIATIONAL FORMUlations of SEMIDEFinite PROBLEMS AND THE METHOD OF SUBSPACE CORRECTIONS}

In a general Hilbert space of infinite dimensions, it is more convenient to discuss the semidefinite problems within a variational framework (without using the notion of unbounded operators). Given a real Hilbert space $V$ with an inner product $(\cdot, \cdot)$ and an induced norm $\|\cdot\|$, we consider a symmetric positive semidefinite bilinear form $a(\cdot, \cdot): V \times V \rightarrow \mathbb{R}$ and the following variational problem: Find $u \in V$ such that

$$
a(u, v)=\langle f, v\rangle, \quad \forall v \in V
$$

for some $f \in V^{*}$. Here $V^{*}$ denotes the space of bounded linear functionals on $V$. We say that the bilinear form $a(\cdot, \cdot)$, is symmetric and positive semidefinite if

$$
a(u, v)=a(v, u), \quad \text { and } \quad a(v, v) \geq 0, \quad \forall u, v \in V .
$$

In what follows, we also use the notation $(\cdot, \cdot)_{a}$ for $a(\cdot, \cdot)$, and we denote the induced seminorm by $|\cdot|_{a}$, that is,

$$
(u, v)_{a}=a(u, v), \quad|u|_{a}=a(u, u)^{1 / 2} .
$$

Associated with the bilinear form $a(\cdot, \cdot)$, we define the following two spaces,

$$
\mathcal{N}=\{v \in V: a(v, w)=0, \quad \forall w \in V\}
$$

and the polar set $\mathcal{N}^{\circ}=\left\{f \in V^{*}:\langle f, v\rangle=0, \quad \forall v \in \mathcal{N}\right\}$.

For the solvability of problem (2.1), we require that $f \in \mathcal{N}^{\circ}$, which is the usual compatibility condition for $f$. In addition, we assume that the bilinear form $a(\cdot, \cdot)$ is continuous and coercive on the quotient space,

$$
\begin{gathered}
a(v, w) \lesssim\|v\|\|w\|, \quad \forall v, w \in V, \\
a(v, v) \gtrsim\|v\|_{V / \mathcal{N}}^{2}, \quad \forall v \in V .
\end{gathered}
$$

The two inequalities (2.2), (2.3) together with the compatibility condition $f \in \mathcal{N}^{\circ}$ will be assumed throughout this paper.

We now introduce an abstract iterative method based on subspace corrections. As a starting point, we split $V$ into a finite sum of closed subspaces, and for such a splitting we assume that

(A0): There exist a finite number of closed subspaces $V_{i} \subset V(i=1, \cdots, J)$ such that

$$
V=\sum_{i=1}^{J} V_{i} .
$$

The assumption (A0) is needed to rule out decompositions such that

$$
V=\overline{\sum_{i=1}^{J} V_{i}}, \quad \text { but } \quad V \neq \sum_{i=1}^{J} V_{i} .
$$


Such a situation may occur when $V$ and some of $V_{i}$ are infinite dimensional, since the sum of closed infinite dimensional subspaces is not necessarily closed (such examples can be found in Deutsch [7] or Rudin [16]). In fact, for decompositions that satisfy (2.5), the convergence of the subspace correction method cannot be guaranteed even for the symmetric and positive definite $a(\cdot, \cdot)$ (see [24]).

Associated with each subspace $V_{i}$, we introduce the local null space $\mathcal{N}_{i}$ as follows:

$$
\mathcal{N}_{i}=\left\{v_{i} \in V_{i}: a\left(v_{i}, w_{i}\right)=0, \quad \forall w_{i} \in V_{i}\right\} .
$$

We note that it is obvious that $\mathcal{N} \cap V_{i} \subset \mathcal{N}_{i}$ and the coercivity condition (2.3) implies the reverse inclusion. Therefore, we have the following equality:

$$
\mathcal{N}_{i}=\mathcal{N} \cap V_{i} .
$$

To define the subspace correction method, we naturally need the solvability of the local problems, which can be guaranteed if conditions analogous to (2.2) and (2.3) are satisfied. In particular, we assume that for each $i=1, \cdots, J$,

(A1): $a\left(v_{i}, v_{i}\right) \gtrsim\left\|v_{i}\right\|_{V_{i} / \mathcal{N}_{i}}^{2}, \quad \forall v_{i} \in V_{i}$.

It is easy to see that (2.2) will hold on each subspace $V_{i}$, since $V_{i} \subset V$. However, (A1), in general does not follow from (2.3) and it needs to be added as an additional assumption (see Example A.1 in Appendix A). We now define the orthogonal complement $\mathcal{N}_{i}^{\perp}$ (as a subspace of $V_{i}$ ) as follows:

$$
\mathcal{N}_{i}^{\perp}=\left\{v_{i} \in V_{i}:\left(v_{i}, w_{i}\right)=0, \quad \forall w_{i} \in \mathcal{N}_{i}\right\} .
$$

Note that $\mathcal{N}_{i}^{\perp}$ is isometrically isomorphic to the quotient space $V_{i} / \mathcal{N}_{i}$. Let $P_{i}$ : $V \mapsto \mathcal{N}_{i}^{\perp}$ be the projection with respect to $a(\cdot, \cdot)$ defined by

$$
a\left(P_{i} v, v_{i}\right)=a\left(v, v_{i}\right), \quad \forall v_{i} \in \mathcal{N}_{i}^{\perp} .
$$

Thanks to $(\mathbf{A} \mathbf{1})$, the operator $P_{i}$ is well-defined. In the algorithm given below, we use inexact projections $T_{i}: V \mapsto \mathcal{N}_{i}^{\perp}$ defined by

$$
T_{i}=R_{i} P_{i}, \quad \forall i=1, \cdots, J,
$$

where $R_{i}: V_{i} \mapsto V_{i}$ are given linear operators. The general subspace correction algorithm is as follows.

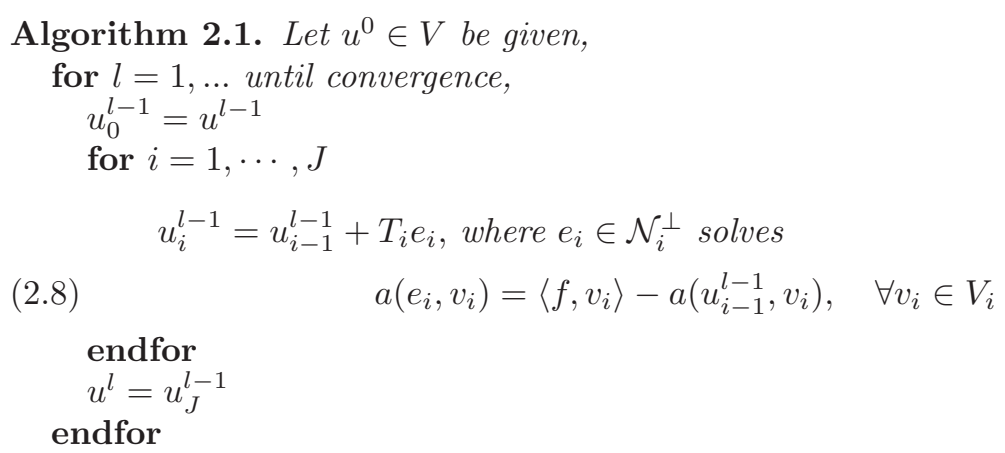

We remark that by the coercivity (A1), the local problem (2.8) is solvable. More importantly, by $T_{i} e_{i}$, we do not mean that the algorithm requires solving the local problem exactly and then applying the operator $T_{i}$ to the exact solution $e_{i}$ of the local problem. Rather, we mean that we correct the current solution $u_{i-1}^{l-1}$ by adding the approximate correction $T_{i} e_{i}$. In particular, if $R_{i}=I$ on $V_{i}$, then the local problem on $V_{i}$ is solved exactly. 
2.1. Relationship with the positive definite case. In this subsection, we shall discuss several technical difficulties that arise in extending the convergence theory for the symmetric and positive definite problems to the case of semidefinite problems. We also point out some similarities between the two cases.

For the symmetric and positive definite problems, the bilinear form $a(\cdot, \cdot)$ can play a role as an inner product $(\cdot, \cdot)$ on the space $V$ and under appropriate assumptions (see 24, Section 4 and Appendix B), the convergence rate identity can be obtained as follows:

$$
\left|\left(I-T_{J}\right) \cdots\left(I-T_{1}\right)\right|_{a}^{2}=1-\frac{1}{K},
$$

where $K=\sup _{|v|_{a}=1} K(v)$ and with $w_{k}:=\sum_{i=k+1}^{J} v_{i}$ and $v_{i} \in V_{i}$,

$$
K(v)=\inf _{\sum v_{k}=v} \sum_{k=1}^{J}\left(\bar{T}_{k}^{-1}\left(v_{k}+T_{k}^{*} w_{k}\right),\left(v_{k}+T_{k}^{*} w_{k}\right)\right)_{a} .
$$

In equation (2.9), the operator $T_{k}^{*}$ is the Hilbert-adjoint of $T_{k}$ with respect to the inner product given by $a(\cdot, \cdot)$ and $\bar{T}_{k}$ is the symmetrization of $T_{k}$,

$$
\bar{T}_{k}=T_{k}+T_{k}^{*}-T_{k}^{*} T_{k} .
$$

A simple observation is that, when $a(\cdot, \cdot)$ is semidefinite, a straightforward definition of the Hilbert-adjoint $T_{k}^{*}$ is not obvious, since $a(\cdot, \cdot)$ is not an inner product. Furthermore, even with an unambiguous definition of $\bar{T}_{k}$, the corresponding operator $\bar{T}_{k}$ does not necessarily have an inverse on $V_{k}$.

In addition, the theory in 24] for the symmetric and positive definite problems, depends crucially on the fact that the operator $I-T_{k}$ is contractive on $V_{k}$ and $\mathcal{R}\left(T_{k}\right)$ and $\mathcal{R}\left(T_{k}^{*}\right)$ are identical, (see Lemma 4.1 in 24 ). The relationships between ranges and null spaces of $T_{k}, T_{k}^{*}$ and $\bar{T}_{k}$ for the semidefinite case are more complicated and the technical results and assumptions that follow are needed to overcome the difficulties related to such "shortcomings". The goal that we would like to achieve, is to obtain the following relation:

$$
K(v)=\inf _{c \in \mathcal{N}} \inf _{\sum} v_{k}=v+c \sum_{k=1}^{J}\left(\bar{T}_{k}^{\dagger}\left(v_{k}+T_{k}^{*} w_{k}\right), v_{k}+T_{k}^{*} w_{k}\right)_{a},
$$

where $w_{k}=\sum_{i=k+1}^{J} v_{i}$ and the operator $\bar{T}_{k}^{\dagger}$ is an appropriate generalization of $\bar{T}_{k}^{-1}$ in (2.9), whose concrete definition is postponed to the next section. We point out here that although (2.10) looks very similar to (2.9), we do not know any obvious way to derive (2.10) from (2.9).

2.2. Some additional assumptions on the subspace solvers. In this subsection we introduce two additional assumptions $(\mathbf{A 2 a})$ and $(\mathbf{A} \mathbf{2 b})$ that are essential for the convergence analysis, outlined in the previous section. We first introduce the adjoint of the subspace solver $T_{i}$ with respect to the bilinear form $a(\cdot, \cdot)$. Thanks to (A1), we can define $R_{i}^{*}: V_{i} \mapsto \mathcal{N}_{i}^{\perp}$ by the following relation:

$$
a\left(R_{i}^{*} w_{i}, v_{i}\right)=a\left(w_{i}, R_{i} v_{i}\right), \quad \forall w_{i} \in V_{i}, v_{i} \in \mathcal{N}_{i}^{\perp} .
$$

Using the operator $R_{i}^{*}$, we define the adjoint $T_{i}^{*}$ as follows:

$$
T_{i}^{*}=R_{i}^{*} P_{i} .
$$


The following important relation is a consequence of definition (2.11):

$$
a\left(T_{i} v, w\right)=a\left(v, T_{i}^{*} w\right), \quad \forall v, w \in V .
$$

Note that this definition is consistent, namely, if $a(\cdot, \cdot)$ is an inner product, then $T_{i}^{*}$ is the Hilbert-adjoint of $T_{i}$ in this inner product. We now introduce $\bar{T}_{i}: V \mapsto V_{i}$, the symmetrization of $T_{i}$ in the usual way,

$$
\bar{T}_{i}=T_{i}+T_{i}^{*}-T_{i}^{*} T_{i}
$$

The following assumptions on $T_{i}$ are needed for analysis later: For each $i=1, \cdots, J$,

(A2a): $\exists \omega_{i} \in(0,2)$ such that $a\left(T_{i} v_{i}, T_{i} v_{i}\right) \leq \omega_{i} a\left(T_{i} v_{i}, v_{i}\right), \quad \forall v_{i} \in V_{i}$,

(A2b): $\exists \beta_{i}>0$ such that $a\left(T_{i} v_{i}, T_{i} v_{i}\right) \geq \beta_{i} a\left(v_{i}, v_{i}\right), \quad \forall v_{i} \in V_{i}$.

These two assumptions are variational analogues of the assumptions provided in 11 and they are necessary and sufficient for the energy norm convergence of the iterates generated by the subspace solvers $T_{i}$ (see also Lemma 2.1 below).

Since $T_{i}=R_{i} P_{i}$, we restate the assumptions $(\mathbf{A} 2 \mathbf{a})$ and $(\mathbf{A} 2 \mathbf{b})$ in a more convenient form, as follows:

(A2a'): $\exists \omega_{i} \in(0,2)$ such that $a\left(R_{i} v_{i}, R_{i} v_{i}\right) \leq \omega_{i} a\left(R_{i} v_{i}, v_{i}\right), \quad \forall v_{i} \in \mathcal{N}_{i}^{\perp}$, (A2b'): $\exists \beta_{i}>0$ such that $a\left(R_{i} v_{i}, R_{i} v_{i}\right) \geq \beta_{i} a\left(v_{i}, v_{i}\right), \quad \forall v_{i} \in \mathcal{N}_{i}^{\perp}$.

2.3. Preliminary results and a generalized inverse of $\bar{T}_{i}$. In this section, we prove several technical results.

Lemma 2.1. Assume that (A1), (A2a) and (A2b) hold, then for each $1 \leq i \leq J$, there exists $\delta_{i} \in[0,1)$ such that

$$
\left|\left(I-T_{i}\right) v_{i}\right|_{a}^{2} \leq \delta_{i}\left|v_{i}\right|_{a}^{2}, \quad \forall v_{i} \in V_{i} .
$$

Proof. For any $v_{i} \in V_{i}$, we have

$$
\begin{aligned}
0 \leq\left|\left(I-T_{i}\right) v_{i}\right|_{a}^{2} & =\left|v_{i}\right|_{a}^{2}-2\left(v_{i}, T_{i} v_{i}\right)_{a}+\left|T_{i} v_{i}\right|_{a}^{2} \\
& \leq\left|v_{i}\right|_{a}^{2}-\frac{2-\omega_{i}}{\omega_{i}}\left|T_{i} v_{i}\right|_{a}^{2} \quad(\text { by }(\mathbf{A 2 a})) \\
& \leq\left(1-\beta_{i} \frac{2-\omega_{i}}{\omega_{i}}\right)\left|v_{i}\right|_{a}^{2} \quad(\text { by }(\mathbf{A 2} \mathbf{b}))
\end{aligned}
$$

which completes the proof.

Note that the proof of Lemma 2.1 shows that the constants $\beta_{i}$ in (A2b) satisfy $\beta_{i} \leq \frac{\omega_{i}}{2-\omega_{i}}$ if (A2a) holds. The following inequalities are direct consequences of the assumptions (A2a), (A2b) and the definitions of $T_{i}, T_{i}^{*}$ and $\bar{T}_{i}$.

Lemma 2.2. Under the assumptions (A2a) and (A2b), the following estimates hold,

$$
\left|T_{i} v_{i}\right|_{a} \gtrsim\left|v_{i}\right|_{a}, \quad\left|T_{i}^{*} v_{i}\right|_{a} \gtrsim\left|v_{i}\right|_{a}, \quad \text { and } \quad\left|\bar{T}_{i} v_{i}\right|_{a} \gtrsim\left|v_{i}\right|_{a} .
$$

The next lemma shows that the null spaces of $T_{i}, T_{i}^{*}$ and $\bar{T}_{i}$ are all the same as $\mathcal{N}_{i}$ when restricted to the subspace $V_{i}$.

Lemma 2.3. Assume that (A1), (A2a) and (A2b) hold. Then we have the following relations:

$$
\mathcal{N} \subset \mathcal{N}\left(T_{i}\right) \cap \mathcal{N}\left(T_{i}^{*}\right) \cap \mathcal{N}\left(\bar{T}_{i}\right)
$$

and

$$
\mathcal{N}\left(T_{i}\right) \cap V_{i}=\mathcal{N}\left(T_{i}^{*}\right) \cap V_{i}=\mathcal{N}\left(\bar{T}_{i}\right) \cap V_{i}=\mathcal{N}_{i} .
$$


Proof. The proofs of (2.14) and (2.15) follow directly from the definitions of $T_{i}, T_{i}^{*}$ and $\bar{T}_{i}$, Lemma 2.2 and assumption (A1).

The result stated in Lemma 2.3 gives an indication on the difference between the study of the semidefinite problems and that of the symmetric and positive definite problems. For the symmetric and positive definite problems 24, we have that $\mathcal{N}\left(T_{i}\right) \cap V_{i}=\mathcal{N}\left(T_{i}^{*}\right) \cap V_{i}=\mathcal{N}\left(\bar{T}_{i}\right) \cap V_{i}=\{0\}$ and hence, $T_{i}: V_{i} \mapsto V_{i}$ is an isomorphism. However, in the current settings, for semidefinite problems, $\mathcal{N}\left(T_{i}\right) \cap V_{i}=\mathcal{N}\left(T_{i}^{*}\right) \cap V_{i}=\mathcal{N}\left(\bar{T}_{i}\right) \cap V_{i}=\mathcal{N}_{i}$, and $\mathcal{N}_{i} \neq\{0\}$ in general. The discussion in the rest of this subsection is devoted to the definition and the properties of an appropriate generalized inverse of the operator $\bar{T}_{i}$.

Lemma 2.4. Under the assumptions (A1), (A2a) and (A2b) for $i=1, \cdots, J$, we have:

Furthermore,

$$
\mathcal{R}\left(\bar{T}_{i}\right) \text { is closed in } V_{i} \text {. }
$$

Proof. The fact that $\bar{T}_{i}$ is closed follows from Lemma 2.2. Now if $\bar{T}_{i} v_{i} \in \mathcal{R}\left(\bar{T}_{i}\right) \cap \mathcal{N}_{i}$, then $\left|\bar{T}_{i} v_{i}\right|_{a}=0$ and, again by Lemma 2.2. $\left|v_{i}\right|_{a}=0 . v_{i} \in \mathcal{N}_{i}$ and hence $\bar{T}_{i} v_{i}=0$. This proves (2.16).

We need a generalized inverse $\bar{T}_{i}^{\dagger}: V \mapsto V_{i}$ satisfying

$$
\bar{T}_{i}^{\dagger} c=0, \quad \forall c \in \mathcal{N} .
$$

From Lemma 2.3 and Lemma 2.4, it follows that $\bar{T}_{i}: \mathcal{N}_{i}^{\perp} \rightarrow \mathcal{R}\left(\bar{T}_{i}\right)$ is an isomorphism, and hence $\bar{T}_{i}^{-1}: \mathcal{R}\left(\bar{T}_{i}\right) \rightarrow \mathcal{N}_{i}^{\perp}$ is well defined. We note that the classical Moore-Penrose generalized inverse, $\bar{T}_{i}^{\times}$of $\bar{T}_{i}$ is a simple zero extension of $\bar{T}_{i}^{-1}$ onto $V_{i}$, (see [8]). More precisely, its definition is as follows:

$$
\begin{aligned}
\bar{T}_{i}^{\times} w & =0, \quad \forall w \in \mathcal{R}\left(\bar{T}_{i}\right)^{\perp}, \\
\bar{T}_{i}^{\times} v & =\bar{T}_{i}^{-1} v, \quad \forall v \in \mathcal{R}\left(\bar{T}_{i}\right) .
\end{aligned}
$$

The classical Moore-Penrose generalized inverse does not necessarily satisfy (2.17) since $\bar{T}_{i}$ is not self-adjoint in the classical sense and hence the Closed Range Theorem cannot be applied to show that $\mathcal{R}\left(\bar{T}_{i}\right)^{\perp}=\mathcal{N}\left(\bar{T}_{i}\right)$. To have the property (2.17) hold true, we introduce $\bar{T}_{i}^{\dagger}$ by

$$
\bar{T}_{i}^{\dagger}=\left(\bar{T}_{i}^{2}\right)^{\times} \bar{T}_{i} .
$$

By Lemma 2.4 and the definition (2.18), the operator $\bar{T}_{i}^{2}: \mathcal{N}_{i}^{\perp} \rightarrow \mathcal{R}\left(\bar{T}_{i}^{2}\right) \subseteq$ $\mathcal{R}\left(\bar{T}_{i}\right)$ is an isomorphism, and $\bar{T}_{i}^{\dagger}=\bar{T}_{i}^{-1}$ on $\mathcal{R}\left(\bar{T}_{i}\right)$. Hence, $\bar{T}_{i}^{\dagger}$ is an extension of $\bar{T}_{i}^{-1}: \mathcal{R}\left(\bar{T}_{i}\right) \rightarrow \mathcal{N}_{i}^{\perp}$ on $V$, satisfying (2.17).

\section{Reduction to the positive definite Case}

One key idea of the convergence analysis of Algorithm 2.1 is to use appropriate restrictions of $a(\cdot, \cdot)$ and the subspace solvers $T_{i}$ 's onto $\mathcal{N}^{\perp}$ and then apply the theory for symmetric and positive definite problems.

We first introduce $Q: V \mapsto \mathcal{N}^{\perp}$, which is the orthogonal projection with respect to the inner product $(\cdot, \cdot)$, defined as

$$
(Q v, w)=(v, w), \quad \forall v \in V, w \in \mathcal{N}^{\perp} .
$$


In order to perform the analysis in the aforementioned direction, for any continuous operator $Y: V \mapsto V$, we define $Y_{a}: V \mapsto V$ by

$$
Y_{a}:=Q Y
$$

and for any closed subspace $W \subset V, W_{a}$ denotes

$$
W_{a}:=\{u \in V \mid u=Q w ; w \in W\}=Q W .
$$

In the case when the operator or the subspace has a subscript, for example, $Y_{i}$ : $V \mapsto V$ and $W_{i} \subset V$, then $Y_{i, a}$ and $W_{i, a}$ denote $Q Y_{i}$ and $Q W_{i}$ respectively.

The following lemma gives some relationships between the subspace solvers and the orthogonal projection $Q$.

Lemma 3.1. Let $Y: V \mapsto V$ be such that $\mathcal{N} \subset \mathcal{N}(Y)$. Then $Y Q=Y$, and in particular,

$$
T_{i} Q=T_{i}, \quad T_{i}^{*} Q=T_{i}^{*}, \quad \text { and } \quad \bar{T}_{i} Q=\bar{T}_{i} .
$$

Next we verify the relevant assumptions that are required by the theory in [24].

Lemma 3.2. Suppose that (A0), (A1), (A2a) and (A2b) hold. Then

(P0): $V_{i, a}$ is closed and $\mathcal{N}^{\perp}=\sum_{i=1}^{J} V_{i, a}$,

(P1): $\exists \omega_{i} \in(0,2)$ such that $a\left(T_{i, a} v_{i}, T_{i, a} v_{i}\right) \leq \omega_{i} a\left(T_{i, a} v_{i}, v_{i}\right), \quad \forall v_{i} \in V_{i, a}$,

(P2): $T_{i, a}: V_{i, a} \mapsto V_{i, a}$ is an isomorphism.

Proof. To prove (P0), we observe that by definition of $Q$ and (A1), the following bound holds:

$$
\left\|Q v_{i}\right\|^{2} \gtrsim a\left(Q v_{i}, Q v_{i}\right)=a\left(v_{i}, v_{i}\right) \gtrsim\left\|v_{i}\right\|_{V_{i} / \mathcal{N}_{i}}^{2}, \quad \forall v_{i} \in V_{i} .
$$

This implies $V_{i, a}=Q V_{i}$ is closed since $V_{i} / \mathcal{N}_{i}$ is closed. Furthermore, we have, by (A0), that

$$
\mathcal{N}^{\perp}=Q V=\sum_{i=1}^{J} Q V_{i}=\sum_{i=1}^{J} V_{i, a} .
$$

This concludes the proof of $(\mathbf{P} \mathbf{0})$.

Next, we observe that for all $v_{i} \in V_{i}$,

$$
\begin{aligned}
a\left(T_{i, a} Q v_{i}, T_{i, a} Q v_{i}\right) & =a\left(T_{i} v_{i}, T_{i} v_{i}\right) \\
& \leq \omega_{i} a\left(T_{i} v_{i}, v_{i}\right) \quad(\text { by }(\mathbf{A 2 a})) \\
& =\omega_{i} a\left(T_{i, a} Q v_{i}, Q v_{i}\right),
\end{aligned}
$$

which gives (P1). It remains to show $(\mathbf{P} 2)$. First, by $(\mathbf{A} 2 \mathbf{b})$, for all $v_{i} \in V_{i}$, we have

$$
a\left(T_{i, a} Q v_{i}, T_{i, a} Q v_{i}\right) \gtrsim a\left(Q v_{i}, Q v_{i}\right),
$$

which shows that $T_{i, a}: V_{i, a} \mapsto V_{i, a}$ is injective and $\mathcal{R}\left(T_{i, a}\right)=T_{i, a}\left(V_{i, a}\right)$ is closed. We now only need to show $\mathcal{R}\left(T_{i, a}\right)=V_{i, a}$. Since $a(\cdot, \cdot)$ is symmetric and positive definite on $V_{i, a}$, we can define $T_{i, a}^{*}: V_{i, a} \mapsto V_{i, a}$, the adjoint of $T_{i, a}: V_{i, a} \mapsto V_{i, a}$, with respect to the inner product $a(\cdot, \cdot)$. By the definition of $T_{i, a}^{*}: V_{i, a} \mapsto V_{i, a}$, we get that $\mathcal{N}\left(T_{i, a}^{*}\right)=\{0\}$. Thus, we may decompose $V_{i, a}$ in the following way:

$$
V_{i, a}=\overline{\mathcal{R}\left(T_{i, a}\right)} \oplus \mathcal{N}\left(T_{i, a}^{*}\right)=\overline{\mathcal{R}\left(T_{i, a}\right)}=\mathcal{R}\left(T_{i, a}\right) .
$$

This completes the proof of (P2) and the lemma. 
Let $u \in V$ be a solution to (2.1) and $\left\{u^{l}: l=0, \cdots\right\}$ be the iterates generated by Algorithm 2.1 respectively. The following relation is standard:

$$
u-u^{l}=E_{J}\left(u-u^{l-1}\right)=\ldots=E_{J}^{l}\left(u-u^{0}\right), \quad E_{J}=\left(I-T_{J}\right) \cdots\left(I-T_{1}\right) .
$$

The convergence rate can be estimated by bounding the following energy norm of $E_{J}$ :

$$
\left|E_{J}\right|_{a}=\sup _{v \in \mathcal{N}^{\perp}} \frac{\left|E_{J} v\right|_{a}}{|v|_{a}} .
$$

By Lemma 3.1 we have

$$
\begin{aligned}
\left|E_{J} v\right|_{a}^{2} & =\left(E_{J} v, E_{J} v\right)_{a} \\
& =\left(E_{J, a} v, E_{J, a} v\right)_{a} \\
& =\left(\left(I-T_{J, a}\right)\left(I-T_{J-1}\right) \cdots\left(I-T_{1}\right) v, E_{J, a} v\right)_{a} \\
& =\left(\left(I-T_{J, a}\right)\left(I-T_{J-1, a}\right) \cdots\left(I-T_{1}\right) v, E_{J, a} v\right)_{a} \\
& =\cdots \\
& =\left(\left(I-T_{J, a}\right)\left(I-T_{J-1, a}\right) \cdots\left(I-T_{1, a}\right) v, E_{J, a} v\right)_{a} \\
& =\left(\left(I-T_{J, a}\right) \cdots\left(I-T_{1, a}\right) v,\left(I-T_{J, a}\right) \cdots\left(I-T_{1, a}\right) v\right)_{a} .
\end{aligned}
$$

Hence

$$
\left|E_{J}\right|_{a}=\sup _{v \in \mathcal{N}^{\perp}} \frac{\left|\left(I-T_{J, a}\right) \cdots\left(I-T_{1, a}\right) v\right|_{a}}{|v|_{a}} .
$$

From the relation (3.2), Lemma 3.2 and the fact that $a(\cdot, \cdot): \mathcal{N}^{\perp} \times \mathcal{N}^{\perp} \mapsto \mathbb{R}$ is symmetric and positive definite, we obtain the following auxiliary result (see [24]):

Lemma 3.3. Suppose that (A0), (A1), (A2a) and (A2b) hold. Then

$$
\left|\left(I-T_{J, a}\right) \cdots\left(I-T_{1, a}\right)\right|_{a}^{2}=1-\frac{1}{K},
$$

where $K=\sup _{|v|_{a}=1} K(v)$ and with $w_{k, a}:=\sum_{i=k+1}^{J} v_{i, a}$ and $v_{i, a} \in V_{i, a}$,

$$
K(v)=\inf _{\sum v_{k, a}=v} \sum_{k=1}^{J}\left(\bar{T}_{k, a}^{-1}\left(v_{k, a}+T_{k, a}^{*} w_{k, a}\right),\left(v_{k, a}+T_{k, a}^{*} w_{k, a}\right)\right)_{a} .
$$

Proof. The proof is provided in Appendix B,

In (3.3), $T_{i, a}^{*}$ and $\bar{T}_{i, a}$ denote the adjoint and the symmetrization of $T_{i, a}: \mathcal{N}^{\perp} \mapsto$ $V_{i, a}$ with respect to the inner product $a(\cdot, \cdot)$, respectively. By the definition of $T_{i, a}^{*}$, we have that

$$
T_{i, a}^{*}=Q T_{i}^{*}
$$

and by Lemma 3.1, we have

$$
\bar{T}_{i, a}=T_{i, a}+T_{i, a}^{*}-T_{i, a}^{*} T_{i, a}=Q\left(T_{i}+T_{i}^{*}-T_{i}^{*} T_{i}\right)=Q \bar{T}_{i} .
$$

Thus, the notation doesn't cause any confusion, and (3.4) and (3.5) will be applied directly in what follows. 


\section{Convergence RAte identities for the MSSC Method}

The main result of this paper is given in the next theorem and it is an expression for $K(v)$ in Lemma 3.3 in terms of $T_{i}, T_{i}^{*}$ and $\bar{T}_{i}$ (which are used in the algorithm), without using projections on $V_{i, a}$.

We first prove a lemma that relates $\bar{T}_{i, a}^{-1}$ and the generalized inverse $\bar{T}_{i}^{\dagger}$ on $V_{i, a}$.

Lemma 4.1. Suppose that (A1), (A2a) and (A2b) hold. Then for each $1 \leq i \leq J$, we have

$$
Q \bar{T}_{i}^{\dagger}=\left(Q \bar{T}_{i}\right)^{-1} \quad \text { on } \quad V_{i, a} .
$$

Proof. For any given $v_{i} \in V_{i}$, assume that $v_{i}=w_{i}+c_{i}$ where $w_{i} \in \mathcal{N}_{i}^{\perp}$ and $c_{i} \in \mathcal{N}_{i}$. Then we have

$$
Q \bar{T}_{i}^{\dagger} Q \bar{T}_{i}\left(Q v_{i}\right)=Q\left(\bar{T}_{i}^{2}\right)^{\times} \bar{T}_{i} Q \bar{T}_{i} v_{i}=Q\left(\bar{T}_{i}^{2}\right)^{\times} \bar{T}_{i} \bar{T}_{i} w_{i}=Q w_{i}=Q v_{i} .
$$

By Lemma 2.2 and Lemma 2.4, $\bar{T}_{i}$ is symmetric and positive definite on $\mathcal{R}\left(\bar{T}_{i}\right)$ with respect to $a(\cdot, \cdot)$. As a direct consequence, $\mathcal{R}\left(\bar{T}_{i}\right)=\mathcal{R}\left(\bar{T}_{i}^{2}\right)$. Then there exists $u_{i} \in \mathcal{N}_{i}^{\perp}$ such that $\bar{T}_{i} v_{i}=\bar{T}_{i}^{2} u_{i}$. By Lemma 2.3, we have $v_{i}-\bar{T}_{i} u_{i} \in \mathcal{N}_{i}$. Finally we get that

$$
Q \bar{T}_{i} Q \bar{T}_{i}^{\dagger}\left(Q v_{i}\right)=Q \bar{T}_{i}\left(\bar{T}_{i}^{2}\right)^{\times} \bar{T}_{i} v_{i}=Q \bar{T}_{i}\left(\bar{T}_{i}^{2}\right)^{\times} \bar{T}_{i}^{2} u_{i}=Q \bar{T}_{i} u_{i}=Q v_{i} .
$$

(4.2) and (4.3) clearly show (4.1).

The following theorem is the main result of this paper.

Theorem 4.1. Under the assumptions, (A0), (A1), (A2a) and (A2b), the $K$ in Lemma 3.3 is given by $K=\sup _{|v|_{a}=1, v \in \mathcal{N} \perp} K(v)$, where

$$
K(v)=\inf _{c \in \mathcal{N}} \inf _{\sum} \sum_{k}=v+c \sum_{k=1}^{J}\left(\bar{T}_{k}^{\dagger}\left(v_{k}+T_{k}^{*} w_{k}\right), v_{k}+T_{k}^{*} w_{k}\right)_{a},
$$

with $w_{k}=\sum_{i=k+1}^{J} v_{i}$

Proof. We first prove the following equality,

$$
\begin{aligned}
& \sum_{k=1}^{J}\left(\bar{T}_{k, a}^{-1}\left(v_{k, a}+T_{k, a}^{*} w_{k, a}\right), v_{k, a}+T_{k, a}^{*} w_{k, a}\right)_{a} \\
= & \sum_{k=1}^{J}\left(\bar{T}_{k}^{\dagger}\left(v_{k}+T_{k}^{*} w_{k}\right),\left(v_{k}+T_{k}^{*} w_{k}\right)\right)_{a},
\end{aligned}
$$

where $v_{k, a}=Q v_{k}$ for some $v_{k} \in V_{k}$ with $k=1, \cdots, J, w_{k, a}=\sum_{i=k+1}^{J} v_{i, a}$ and $w_{k}=\sum_{i=k+1}^{J} v_{i}$.

Observe that with $\chi_{k, a}=v_{k, a}+T_{k, a}^{*} w_{k, a} \in V_{k, a}$,

$$
\begin{aligned}
\left(\bar{T}_{k, a}^{-1} \chi_{k, a}, \chi_{k, a}\right)_{a} & =\left(\left(Q \bar{T}_{k}\right)^{-1} \chi_{k, a}, \chi_{k, a}\right)_{a} \\
& =\left(Q \bar{T}_{k}^{\dagger} \chi_{k, a}, \chi_{k, a}\right)_{a} \quad(\text { by Lemma 4.1) } \\
& =\left(\bar{T}_{k}^{\dagger} \chi_{k, a}, \chi_{k, a}\right)_{a} \\
& =\left(\bar{T}_{k}^{\dagger}\left(Q v_{k}+Q T_{k}^{*} w_{k}\right),\left(Q v_{k}+Q T_{k}^{*} w_{k}\right)\right)_{a} \\
& =\left(\bar{T}_{k}^{\dagger}\left(v_{k}+T_{k}^{*} w_{k}\right),\left(v_{k}+T_{k}^{*} w_{k}\right)\right)_{a} .
\end{aligned}
$$


In the last two equalities above, we used Lemma 2.3 and the property of $\bar{T}_{i}^{\dagger}$ that $\bar{T}_{i}^{\dagger} c=0$ for all $c \in \mathcal{N}$. Equation (4.4) then follows from the above equalities.

Thanks to Lemma 3.2 and Lemma 3.3. for $K(v)$ we have

$$
K(v)=\inf _{\sum v_{k, a}=v} \sum_{k=1}^{J}\left(\bar{T}_{k, a}^{-1}\left(v_{k, a}+T_{k, a}^{*} w_{k, a}\right), v_{k, a}+T_{k, a}^{*} w_{k, a}\right)_{a} .
$$

To complete the proof, we need to show that $K(v)$ defined in (4.5) is equal to the following quantity:

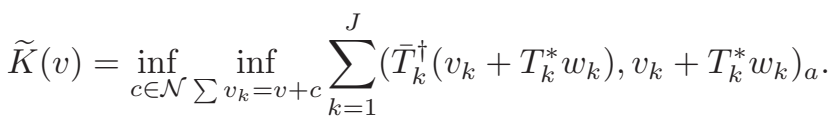

We first show $\widetilde{K}(v) \leq K(v)$. Let $\left\{v_{k, a}\right\}, v_{k, a} \in V_{k, a}$ be a decomposition satisfying $\sum_{k=1}^{J} v_{k, a}=v$. Such a decomposition exists because $\mathcal{N}^{\perp}=\sum_{k=1}^{J} V_{k, a}$. By the definition of $V_{k, a}$, there exist $v_{k} \in V_{k}$ such that $Q v_{k}=v_{k, a}$ for all $k=1, \ldots, J$. Moreover, we have that $c=\sum_{k=1}^{J}\left(v_{k}-v_{k, a}\right) \in \mathcal{N}$. Applying (4.4) for the decompositions $\left\{v_{k, a}\right\}$ and $\left\{v_{k}\right\}$, together with the definition of $\widetilde{K}(v)$, gives that

$$
\begin{aligned}
\widetilde{K}(v) & \leq \sum_{k=1}^{J}\left(\bar{T}_{k}^{\dagger}\left(v_{k}+T_{k}^{*} w_{k}\right), v_{k}+T_{k}^{*} w_{k}\right)_{a} \\
& =\sum_{k=1}^{J}\left(\bar{T}_{k, a}^{-1}\left(v_{k, a}+T_{k, a}^{*} w_{k, a}\right), v_{k, a}+T_{k, a}^{*} w_{k, a}\right)_{a} .
\end{aligned}
$$

Since the decomposition $\left\{v_{k, a}\right\}$ of $v$ is arbitrary, we have

$$
\widetilde{K}(v) \leq \inf _{\sum_{k=1}^{J} v_{k, a}=v} \sum_{k=1}^{J}\left(\bar{T}_{k, a}^{-1}\left(v_{k, a}+T_{k, a}^{*} w_{k, a}\right), v_{k, a}+T_{k, a}^{*} w_{k, a}\right)_{a}=K(v) .
$$

To show the reverse inequality $K(v) \leq \widetilde{K}(v)$ we use a similar argument. Let $c \in \mathcal{N}$ and $v \in \mathcal{N}^{\perp}$ be fixed. Then by (A0), there exists a decomposition $\left\{v_{k}\right\}_{k=1}^{J}$ such that $\sum_{k=1}^{J} v_{k}=v+c$. Now we define $v_{k, a}=Q v_{k}$ and observe that this implies $\sum_{k=1}^{J} v_{k, a}=v$. Again, from (4.4), we have

$$
K(v) \leq \sum_{k=1}^{J}\left(\bar{T}_{k}^{\dagger}\left(v_{k}+T_{k}^{*} w_{k}\right), v_{k}+T_{k}^{*} w_{k}\right)_{a} .
$$

Since $\left\{v_{k}\right\}$ can be any decomposition of $v+c$, we may take an infimum over all such decompositions, to obtain that

$$
K(v) \leq \inf _{\sum_{k=1}^{J} v_{k}=v+c}\left(\bar{T}_{k}^{\dagger}\left(v_{k}+T_{k}^{*} w_{k}\right), v_{k}+T_{k}^{*} w_{k}\right)_{a} .
$$

Observe that the left side of the above inequality is independent of $c \in \mathcal{N}$. Then we conclude the proof by taking infimum over $c \in \mathcal{N}$ on the right side of (4.7).

If $T_{i}=P_{i}$ for all $i$, namely, only exact subspace solvers are employed, which is an important special case, we have the following result. 
Corollary 4.1. Assume $T_{i}=P_{i}$ for $i=1, \cdots, J$. Then under the assumptions (A0) and (A1) the following equality holds:

$$
K=\sup _{|v|_{a}=1, v \in \mathcal{N}^{\perp}} \inf _{c \in \mathcal{N}} \inf _{\sum v_{k}=v+c} \sum_{k=1}^{J}\left(P_{k} \sum_{i=k}^{J} v_{i}, \sum_{i=k}^{J} v_{i}\right)_{a} .
$$

This result will be used for the analysis of multigrid method with Gauss-Seidel smoothing in Section 5 .

In passing to the main application of our theory for the multigrid methods, we shall make a simple illustration by considering the following linear system of equations:

$$
A u=f,
$$

where $A \in \mathbb{R}^{n \times n}$ is symmetric and a positive semidefinite matrix with positive diagonal elements, and $f \in \mathbb{R}^{n}$ is assumed to belong to the range of $A$ for consistency.

We now apply the abstract results to the Gauss-Seidel method for (4.8). It is well known, that the Gauss-Seidel method corresponds to a matrix splitting $A=M-N$ with

$$
M=D-L \quad \text { and } \quad N=L^{t},
$$

where $D$ is the diagonal, $-L$ is the strictly lower triangular part of $A$, and $L^{t}$ denotes the transpose of $L$. In this case, the error propagation matrix $E$ is given by

$$
E=\left(I-(D-L)^{-1} A\right)=\left(I-P_{n}\right) \cdots\left(I-P_{1}\right),
$$

where $P_{i}=\frac{\left(A e_{i}, \cdot\right)}{\left(A e_{i}, e_{i}\right)} e_{i}$ with $\left\{e_{1}, \cdots, e_{n}\right\}$ being the canonical basis for $\mathbb{R}^{n}$.

The energy norm convergence rate is then given by

$$
|E|_{A}=\left(\sup _{v \in \mathcal{N}(A)^{\perp}} \frac{(E v, E v)_{A}}{(v, v)_{A}}\right)^{1 / 2},
$$

where $(\cdot, \cdot)_{A}=(A \cdot, \cdot)$ with $(\cdot, \cdot)=(\cdot, \cdot)_{\ell^{2}}$ being the usual $\ell^{2}$ inner product. Since $D$ is assumed to be positive definite, a simple application of Theorem 4.1, leads to the following convergence result.

Corollary 4.2. If $A$ is symmetric and positive semidefinite with positive diagonal, then the energy norm convergence rate given by $|E|_{A}$ of the Gauss-Seidel iterative method is given as follows:

$$
|E|_{A}^{2}=1-K^{-1},
$$

where with $S=L D^{-1} L^{T}$,

$$
K=1+\sup _{v \in \mathcal{N}(A) \perp} \inf _{c \in \mathcal{N}(A)} \frac{(S(v+c),(v+c))}{(v, v)_{A}} .
$$

\section{Multigrid method For Neumann problems}

In this section we apply Theorem 4.1 to obtain a convergence result for a multigrid method applied to a pure Neumann boundary value problem. One main idea in our analysis is to estimate $K(v)$ by taking special decomposition of the finite element space on the finest grid into a sum of one-dimensional subspaces spanned by nodal basis functions on all levels. 
We consider the following Neumann boundary problem:

$$
-\Delta u=f \quad \text { in } \Omega, \quad \frac{\partial u}{\partial \mathbf{n}}=0 \quad \text { on } \partial \Omega,
$$

where $\frac{\partial u}{\partial \mathbf{n}}$ is the exterior normal derivative of $u$ and $\Omega$ is a polygonal domain in $\mathbb{R}^{d}$ with $d=1,2$ or 3 . The variational problem corresponding to (5.1) can be given as follows: Find $u \in H^{1}(\Omega)$ such that

$$
a(u, v)=\langle f, v\rangle, \quad \forall v \in H^{1}(\Omega),
$$

where

$$
a(u, v)=\int_{\Omega} \nabla u \cdot \nabla v d x \quad \text { and } \quad\langle f, v\rangle=\int_{\Omega} f v d x .
$$

The null space $\mathcal{N}$ of $a(\cdot, \cdot)$ is given by

$$
\mathcal{N}=\operatorname{span}\{1\}
$$

and it is well-known that for the solvability of (5.2), it is necessary that $f$ satisfies the following compatibility condition:

$$
\int_{\Omega} f d x=0
$$

It is then easy to see that the solution to (5.1) exists and is unique (in the weak sense) on the quotient space $H^{1}(\Omega) / \mathcal{N}$.

Throughout this section, we also assume that $\Omega$ is triangulated with a nested sequence of quasi-uniform triangulations $\mathcal{T}_{k}=\left\{\tau_{k}^{i}\right\}$. As usual, the mesh size is denoted by $h_{k}$, and we assume that the quasi-uniformity constants are independent of $k$ and $h_{k} \sim \gamma^{k}$ with $\gamma \in(0,1)$, for $k=1, \cdots, L$. Associated with each $\mathcal{T}_{k}$, we have the finite element space of continuous piecewise linear functions $V_{k} \subset H^{1}(\Omega)$. We then obtain a finite number of nested spaces,

$$
V_{1} \subset \cdots \subset V_{k} \subset \cdots \subset V_{L} .
$$

As a basis in $V_{k}$ we choose the standard nodal Courant basis functions $\phi_{k}^{i}$, namely

$$
V_{k}=\operatorname{span}\left\{\phi_{k}^{1}, \cdots, \phi_{k}^{n_{k}}\right\}=\sum_{i=1}^{n_{k}} V_{k}^{i},
$$

where $V_{k}^{i}=\operatorname{span}\left\{\phi_{k}^{i}\right\}$ and each $\phi_{k}^{i}$ is the usual finite element basis function, that is, one at the node $x_{k}^{i}$ and zero at the other nodes and $n_{k}$ is the dimension of $V_{k}$, or the number of grid nodes, $\left\{x_{k}^{j}\right\}_{j=1}^{n_{k}}$ for $V_{k}$.

We are interested in solving the following equations resulting from the finite element discretization (with an abuse of notation): find $u \in V$ with $h=h_{L}$ and $V=V_{L}$ such that

$$
a(u, v)=\langle f, v\rangle, \quad \forall v \in V .
$$

To solve problem (5.3), we first decompose the space $V$ in the following manner:

$$
V=\sum_{k=1}^{L} \sum_{i=1}^{n_{k}} V_{k}^{i}
$$

Under the above settings, we observe that the subspace correction method based on the subspace decomposition (5.4) with exact subspace (local) solver for each subspace $V_{k}^{j}$ for $k=1, \cdots, L$ and $j=1, \cdots, n_{k}$ can be understood as the wellknown "/" multigrid algorithm with one Gauss-Seidel sweep in each subspace $V_{k}$ 
for $k=1, \cdots, L$, (see 25]). For such a multigrid method, Theorem 4.1 can be applied, since the error transfer operator $E$ for such a method can be written as a product of nonexpansive operators, namely

$$
E=\prod_{k=1}^{L} \prod_{j=1}^{n_{k}}\left(I-P_{k}^{j}\right)
$$

where $P_{k}^{l}$ is the exact projection onto the one-dimensional space $V_{k}^{l}$, (see also [25]). By a direct application of Corollary 4.1, we obtain that

$$
|E|_{a}^{2}=1-K^{-1},
$$

where

$$
K=\sup _{v \in \mathcal{N}^{\perp}} \inf _{c \in \mathcal{N}} \inf _{\sum_{k=1}^{L} \sum_{i=1}^{n_{k}} v_{k}^{i}=v+c} \frac{\sum_{k=1}^{L} \sum_{i=1}^{n_{k}}\left|P_{k}^{i}\left(\sum_{(l, j) \geq(k, i)} v_{l}^{j}\right)\right|_{a}^{2}}{(v, v)_{a}}
$$

where $n_{k}$ is the number of nodal points in each subspace $V_{k}$ with $k=1, \cdots, L$ and

$$
\sum_{(l, j) \geq(k, i)} v_{l}^{j}=\sum_{j=i}^{n_{k}} v_{k}^{j}+\sum_{l=k+1}^{L} \sum_{j=1}^{n_{l}} v_{l}^{j} .
$$

The next theorem, which is the main result of this section, gives a uniform bound on the constant $K$, and thus of the norm $|E|_{a}$.

Theorem 5.1. The norm of the error transfer operator, given by (5.5) is bounded as follows:

$$
|E|_{a}^{2} \leq \delta<1,
$$

with $\delta$ independent of the mesh size $h$ and the number of levels $L$.

Proof. Given $v \in \mathcal{N}^{\perp}$, we consider the following decomposition:

$$
v=\sum_{k=1}^{L} v_{k}=\sum_{k=1}^{L} \sum_{i=1}^{n_{k}} v_{k}^{i}, \quad v_{k}^{i} \in V_{k}^{i},
$$

where

$$
v_{k}=\sum_{i=1}^{n_{k}} v_{k}^{i}=\left(Q_{k}-Q_{k-1}\right) v
$$

and $Q_{k}$ is the $L^{2}$ projection onto $V_{k}$ and $Q_{0}$ is the $L^{2}$ projection onto $\operatorname{span}\{1\}$. Note that $Q_{L} v=v, Q_{0} v=0$ and

$$
\sum_{(l, j) \geq(k, i)} v_{l}^{j}=\sum_{j=i}^{n_{k}} v_{k}^{j}+\sum_{l=k+1}^{L} \sum_{j=1}^{n_{l}} v_{l}^{j}=\sum_{j=i}^{n_{k}} v_{k}^{j}+v-Q_{k} v .
$$


Since for all $w \in V$, and for all $v_{k}^{i} \in V_{k}^{i}$, we have

$$
a\left(P_{k}^{i} P_{k} w, v_{k}^{i}\right)=a\left(P_{k} w, v_{k}^{i}\right)=a\left(w, v_{k}^{i}\right)=a\left(P_{k}^{i} w, v_{k}^{i}\right),
$$

and $a(\cdot, \cdot)$ is $V_{k}^{i}$-elliptic, it follows that $P_{k}^{i} P_{k} w=P_{k}^{i} w \quad \forall w \in V$. Hence

$$
\begin{aligned}
P_{k}^{i} \sum_{(l, j) \geq(k, i)} v_{l}^{j} & =P_{k}^{i} \sum_{j=i}^{n_{k}} v_{k}^{j}+P_{k}^{i}\left(v-Q_{k} v\right) \\
& =P_{k}^{i} \sum_{j=i}^{n_{k}} v_{k}^{j}+P_{k}^{i} P_{k}\left(v-Q_{k} v\right) \\
& =P_{k}^{i} \sum_{j=i}^{n_{k}} v_{k}^{j}+P_{k}^{i}\left(P_{k} v-Q_{k} v\right) .
\end{aligned}
$$

This then yields (with $\Omega_{k}^{i}=\operatorname{supp} \phi_{k}^{i}$ )

$$
\begin{aligned}
\sum_{i=1}^{n_{k}}\left|P_{k}^{i} \sum_{(l, j) \geq(k, i)} v_{l}^{j}\right|_{a}^{2} & =\sum_{i=1}^{n_{k}}\left|P_{k}^{i} \sum_{j=i}^{n_{k}} v_{k}^{j}+P_{k}^{i}\left(P_{k} v-Q_{k} v\right)\right|_{a}^{2} \\
& \lesssim\left(\sum_{i=1}^{n_{k}}\left|P_{k}^{i} \sum_{j=i}^{n_{k}} v_{k}^{j}\right|_{a}^{2}+\sum_{i=1}^{n_{k}}\left|P_{k}^{i}\left(P_{k} v-Q_{k} v\right)\right|_{a}^{2}\right) \\
& \lesssim\left(\sum_{i=1}^{n_{k}} \sum_{j \in N_{k}(i)}\left|v_{k}^{j}\right|_{a, \Omega_{k}^{i}}^{2}+\sum_{i=1}^{n_{k}}\left|\left(P_{k} v-Q_{k} v\right)\right|_{a, \Omega_{k}^{i}}^{2}\right)
\end{aligned}
$$

where $N_{k}(i)=\left\{j \in\left\{1, \cdots, n_{k}\right\}: \Omega_{k}^{j} \cap \Omega_{k}^{i} \neq \emptyset\right\}$. Now by $\left(Q_{k}-Q_{k-1}\right)^{2}=\left(Q_{k}-\right.$ $\left.Q_{k-1}\right)$ and a standard error estimate for $\left\|\left(Q_{k}-Q_{k-1}\right) v\right\|_{0}^{2}$ it follows that

$$
\begin{aligned}
\sum_{i=1}^{n_{k}} \sum_{j \in N_{k}(i)}\left|v_{k}^{j}\right|_{a, \Omega_{k}^{i}}^{2} & \lesssim \sum_{i=1}^{n_{k}} \sum_{j \in N_{k}(i)}\left|v_{k}\left(x_{k}^{j}\right)\right|^{2} h_{k}^{d-2} \lesssim h_{k}^{-2} \sum_{i=1}^{n_{k}} h_{k}^{d}\left|v_{k}\left(x_{k}^{j}\right)\right|^{2} \\
& \lesssim h_{k}^{-2}\left\|v_{k}\right\|_{0}^{2} \lesssim h_{k}^{-2}\left\|\left(Q_{k}-Q_{k-1}\right) v_{k}\right\|_{0}^{2} \\
& \lesssim h_{k}^{-2} h_{k-1}^{2}\left|v_{k}\right|_{a}^{2} \lesssim \gamma^{-2}\left|v_{k}\right|_{a}^{2},
\end{aligned}
$$

where we have used that $h_{k} \sim \gamma^{k}$ with $\gamma \in(0,1)$. Hence

$$
\begin{aligned}
\sum_{i=1}^{n_{k}}\left|P_{k}^{i} \sum_{(l, j)>(k, i)} v_{l}^{j}\right|_{a}^{2} & \lesssim\left(\left|v_{k}\right|_{a}^{2}+\left|\left(P_{k}-Q_{k}\right) v\right|_{a}^{2}\right) \\
& \lesssim\left(\left|\left(Q_{k}-Q_{k-1}\right) v\right|_{a}^{2}+\left|\left(P_{k}-Q_{k}\right) v\right|_{a}^{2}\right)
\end{aligned}
$$

The proof is completed by applying the following well-known estimates (see Bramble and Zhang [4] or Xu [25]):

$$
\sum_{k=1}^{L}\left(\left|\left(Q_{k}-Q_{k-1}\right) v\right|_{a}^{2}+\left|\left(P_{k} v-Q_{k} v\right)\right|_{a}^{2}\right) \lesssim|v|_{a}^{2}, \quad \forall v \in H^{1}(\Omega) .
$$




\section{Concluding REMARKS}

In this paper, we have established a sharp convergence estimate for the successive subspace correction methods applied to the symmetric and positive semidefinite (singular) problems. As an illustration, using our abstract theory, we have shown that the multigrid method with the Gauss-Seidel smoothing applied to the Laplace equation with pure Neumann boundary conditions converges at an optimal rate independent of the mesh parameter and also the number of levels. Our new multigrid analysis presented in this paper has led to a very transparent convergence rate estimate and it can also be applied to many other elliptic problems.

\section{Appendix A. Some examples}

In this appendix, we provide several examples to clarify our assertions made in the paper.

Example A.1 (Assumption (A1) is necessary in general). We consider $V=\ell^{2}$ (the Hilbert space of all square summable sequences). Let $\left\{e_{1}, e_{2} \cdots\right\}$ be the canonical Euclidean basis in it, i.e. $\left(e_{i}\right)_{j}=\delta_{i j}$, where $\delta_{i j}$ is the Kronecker delta function. We introduce a bilinear form $a(\cdot, \cdot)$ defined by the following equations:

$$
a\left(e_{2 i-1}, e_{2 j-1}\right)=0 \quad \text { and } \quad a\left(e_{2 i}, e_{2 j}\right)=\delta_{i j}, \quad \forall i, j=1, \cdots .
$$

By definition,

$$
\mathcal{N}=\overline{\operatorname{span}\left\{e_{2 i-1}: i=1, \cdots\right\}} \text { and } \quad \mathcal{N}^{\perp}=\overline{\operatorname{span}\left\{e_{2 i}: i=1, \cdots\right\}} .
$$

We take the subspaces $V_{1} \subset V$ to be as follows:

$$
V_{1}=\overline{\operatorname{span}\left\{w_{1}, \cdots w_{i} \cdots\right\}}, \quad w_{i}=\alpha_{i}\left(e_{2 i-1}+\frac{1}{2^{i}} e_{2 i}\right) .
$$

Here $\alpha_{i}=\frac{1}{\sqrt{1+\frac{1}{4^{i}}}} \lesssim 1$ are chosen so that $\left\|w_{i}\right\|=1$. Hence $a\left(w_{i}, w_{j}\right)=\delta_{i j} \alpha_{i} \alpha_{j} 2^{-(i+j)}$ for all $w_{i} \in V_{1}$. On the other hand, we have that $\left\|w_{i}\right\|_{V_{1} / \mathcal{N}_{1}}=\left\|w_{i}\right\|=1$, since $\mathcal{N}_{1}=V_{1} \cap \mathcal{N}=\{0\}$. Thus, for the bilinear form $a(\cdot, \cdot)$ defined by (A.1), the assumption (A1) does not hold, although the coercivity condition (2.3) holds.

Finally, we provide an example that shows although $W \subset V$ is a closed subspace, $Q(W)$ may not necessarily be closed, hence assumption (A1) is necessary in general.

Example A.2. For convenience, we shall use the same notation used in Example A.1. We observe that

$$
Q w_{i}=\frac{1}{\sqrt{1+4^{i}}} e_{2 i}
$$

Hence, $\overline{Q(W)}=\mathcal{N}^{\perp}$. Namely, $Q(W)$ is dense in $\mathcal{N}^{\perp}$. We shall now show that there exists $\breve{w} \in \mathcal{N}^{\perp}$ for which no pre-image in $W$ of $Q$ exists. Namely, $Q: W \mapsto \mathcal{N}^{\perp}$ is not surjective and so $Q(W)$ is not closed. Let us choose $\breve{w} \in \mathcal{N}^{\perp}$ as follows:

$$
\breve{w}=\{0,1,0,1 / 2,0,1 / 4,0, \cdots\}=\sum_{i=1} 2^{-i} e_{2 i}
$$

and assume there exists $w \in W$ such that $Q w=\breve{w}$. It is clear then that $w$ should be of the following form:

$$
w=\sum_{i=1} \mu_{i} w_{i}=\sum_{i=1} \mu_{i} \alpha_{i}\left(e_{2 i-1}+2^{-i} e_{2 i}\right)=\sum_{i=1}\left(e_{2 i-1}+2^{-i} e_{2 i}\right) .
$$


This gives that $\mu_{i}=\alpha_{i}^{-1}$ and as a consequence we obtain that the norm of $w$ grows unboundedly, since

$$
\|w\|^{2}=\sum_{i=1}\left(1+4^{-i}\right)=\infty
$$

and hence we are led to a contradiction.

\section{Appendix B. Proof of Lemma 3.3}

In this appendix, we prove Lemma 3.3 First, we state two lemmas, Lemma B.1 and Lemma B.2 without proof since they can be found in 24. Second, using the lemmas and Theorem 4.2 in [24, we obtain our main auxiliary result, Lemma 3.3 .

Through this appendix $(\cdot, \cdot)_{A}$ is a symmetric and positive definite inner product.

Lemma B.1. The following identity holds, for $k=1, \cdots, J$ :

$$
I+T_{k} \bar{T}_{k}^{-1} T_{k}^{*}\left(I-T_{k}^{-1}\right)=T_{k} \bar{T}_{k}^{-1} .
$$

Lemma B.2. The following identity holds, for $k=1, \cdots, J$ :

$$
\left(T_{k}^{*-1}-I\right) T_{k} \bar{T}_{k}^{-1} T_{k}^{*}\left(T_{k}^{-1}-I\right)=\bar{T}_{k}^{-1}-I .
$$

Finally, the next lemma uses the above two results and Theorem 4.2 in 24] to obtain Lemma 3.3 .

Lemma B.3. Let $v \in V, \sum_{k=1}^{J} v_{k}=v$ and $w_{k}=\sum_{i=k+1}^{J} v_{i}$. For

$$
c_{0}(v)=\sum_{k=1}^{J}\left(T_{k} \bar{T}_{k}^{-1} T_{k}^{*}\left(w_{k}+\left(I-T_{k}^{-1}\right) v_{k}\right), w_{k}+\left(I-T_{k}^{-1}\right) v_{k}\right)_{A},
$$

and

$$
K(v)=\left\{\left(\sum_{k=1}^{J}\left(\bar{T}_{k}^{-1}\left(v_{k}+T_{k}^{*} w_{k}\right),\left(v_{k}+T_{k}^{*} w_{k}\right)\right)_{A}\right\},\right.
$$

one has

$$
K(v)=\|v\|_{A}^{2}+c_{0}(v) .
$$

Proof. A straightforward calculation gives

$$
\begin{aligned}
& (v, v)_{A}+\sum_{k=1}^{J}\left(T_{k} \bar{T}_{k}^{-1} T_{k}^{*}\left(w_{k}+\left(I-T_{k}^{-1}\right) v_{k}\right), w_{k}+\left(I-T_{k}^{-1}\right) v_{k}\right)_{A} \\
= & \left(\sum_{k=1}^{J} v_{k}, \sum_{k=1}^{J} v_{k}\right)_{A} \\
& +\sum_{k=1}^{J}\left(T_{k} \bar{T}_{k}^{-1} T_{k}^{*}\left(w_{k}+\left(I-T_{k}^{-1}\right) v_{k}\right), w_{k}+\left(I-T_{k}^{-1}\right) v_{k}\right)_{A} \\
= & \sum_{k=1}^{J}\left[\left(v_{k}, v_{k}\right)_{A}+2\left(v_{k}, w_{k}\right)_{A}\right. \\
& \left.+\left(T_{k} \bar{T}_{k}^{-1} T_{k}^{*}\left(w_{k}+\left(I-T_{k}^{-1}\right) v_{k}\right), w_{k}+\left(I-T_{k}^{-1}\right) v_{k}\right)_{A}\right] .
\end{aligned}
$$


For each term in the sum above we have

$$
\begin{aligned}
& \left(v_{k}, v_{k}\right)_{A}+2\left(v_{k}, w_{k}\right)_{A}+\left(T_{k} \bar{T}_{k}^{-1} T_{k}^{*}\left(w_{k}+\left(I-T_{k}^{-1}\right) v_{k}\right), w_{k}+\left(I-T_{k}^{-1}\right) v_{k}\right)_{A} \\
& =\left(v_{k}, v_{k}\right)_{A}+\left(T_{k} \bar{T}_{k}^{-1} T_{k}^{*}\left(I-T_{k}^{-1}\right) v_{k},\left(I-T_{k}^{-1}\right) v_{k}\right)_{A} \\
& \quad+2\left[\left(v_{k}, w_{k}\right)_{A}+\left(T_{k} \bar{T}_{k}^{-1} T_{k}^{*}\left(I-T_{k}^{-1}\right) v_{k}, w_{k}\right)_{A}\right]+\left(T_{k} \bar{T}_{k}^{-1} T_{k}^{*} w_{k}, w_{k}\right)_{A} .
\end{aligned}
$$

We use Lemma B.1 and Lemma B.2 to obtain that

$$
\begin{aligned}
& \left(v_{k}, v_{k}\right)_{A}+2\left(v_{k}, w_{k}\right)_{A}+\left(T_{k} \bar{T}_{k}^{-1} T_{k}^{*}\left(w_{k}+\left(I-T_{k}^{-1}\right) v_{k}\right), w_{k}+\left(I-T_{k}^{-1}\right) v_{k}\right)_{A} \\
& =\left(\bar{T}_{k}^{-1} v_{k}, v_{k}\right)_{A}+2\left(\left(I+T_{k} \bar{T}_{k}^{-1} T_{k}^{*}\left(I-T_{k}^{-1}\right)\right) v_{k}, w_{k}\right)_{A}+\left(\bar{T}_{k}^{-1} T_{k}^{*} w_{k}, T_{k}^{*} w_{k}\right)_{A} \\
& =\left(\bar{T}_{k}^{-1} v_{k}, v_{k}\right)_{A}+2\left(T_{k} \bar{T}_{k}^{-1} v_{k}, w_{k}\right)_{A}+\left(\bar{T}_{k}^{-1} T_{k}^{*} w_{k}, T_{k}^{*} w_{k}\right)_{A} .
\end{aligned}
$$

Finally,

$$
\begin{aligned}
(v, v)_{A}+c_{0}(v)= & \sum_{k=1}^{J}\left(\bar{T}_{k}^{-1} v_{k}, v_{k}\right)_{A}+2 \sum_{k=1}^{J}\left(\bar{T}_{k}^{-1} v_{k}, T_{k}^{*} w_{k}\right)_{A} \\
& +\sum_{k=1}^{J}\left(\bar{T}_{k}^{-1} T_{k}^{*} w_{k}, T_{k}^{*} w_{k}\right)_{A} \\
= & \sum_{k=1}^{J}\left(\bar{T}_{k}^{-1}\left(v_{k}+T_{k}^{*} w_{k}\right), v_{k}+T_{k}^{*} w_{k}\right)_{A} .
\end{aligned}
$$

\section{ACKNOWLEDGMENTS}

The authors wish to thank the anonymous referees for their helpful remarks and suggestions.

\section{REFERENCES}

[1] I. Babuška and J. M. Melenk. The partition of unity method. Internat. J. Numer. Methods Engrg., 40(4):727-758, 1997. MR1429534(97j:73071)

[2] A. B. Berman and R. J. Plemmons. Nonnegative matrices in the mathematical sciences. SIAM classics in Applied Mathematics, Philadelphia, 1994. MR1298430 (95e:15013)

[3] Pavel Bochev and R. B. Lehoucq. On the finite element solution of the pure Neumann problem. SIAM Rev., 47(1):50-66 (electronic), 2005. MR2149101(2007a:65185)

[4] J. H. Bramble and X. Zhang. The analysis of multigrid methods. Handbook of numerical analysis, Vol. VII. North-Holland, Amsterdam, 2000. MR1804746(2001m:65183)

[5] J. H. Bramble and J. E. Pasciak. Iterative techniques for time dependent stokes problems. Comput. Math. with Appl., 33:13-30, 1997. MR1442058 (98e:65091)

[6] Z. H. Cao. A note on properties of splittings of singular symmetric positive semidefinite matrices. Numer. Math., 88:603-606, 2001. MR 1836872(2002b:65045)

[7] Frank Deutsch. Best approximation in inner product spaces. CMS Books in Mathematics/Ouvrages de Mathématiques de la SMC, 7. Springer-Verlag, New York, 2001. MR 1823556 (2002c:41001)

[8] Heinz W. Engl, Martin Hanke, and Andreas Neubauer. Regularization of inverse problems, volume 375 of Mathematics and its Applications. Kluwer Academic Publishers Group, Dordrecht, 1996. MR $1408680(97 \mathrm{k}: 65145)$

[9] Wolfgang Hackbusch. Multigrid methods and applications, volume 4 of Springer Series in Computational Mathematics. Springer-Verlag, Berlin, 1985. MR814495 (87e:65082)

[10] H. B. Keller. On the solution of singular and semidefinite linear systems by iteration. J. Soc. Indust. Appl. Math. Ser. B Numer. Anal., 2:281-290, 1965. MR0195244(33:3447)

[11] Young-Ju Lee, Jinbiao Wu, Jinchao Xu, and Ludmil Zikatanov. On the convergence of iterative methods for semidefinite linear systems. SIAM Journal on Matrix Analysis and Applications., 28 (3): 634-641, 2006. MR2262973 
[12] I. Marek and D. B. Szyld. Comparison theorems for the convergence factor of iterative methods for singular matrices. Linear Algebra and its Applications., 316:67-87, 2000. MR.1782417 (2001h:65040)

[13] I. Marek and D. B. Szyld. Algebraic Schwarz methods for the numerical solution of Markov chains. Linear Algebra and its Applications., 386:67-81 Sp. Iss. SI, 2004. MR2066608 (2005i:60005)

[14] R. Nabben and D. B. Szyld. Schwarz iterations for symmetric positive semidefinite problems. SIAM J. Matrix Anal. Appl., 29:98-116. MR2288016

[15] S. V. Nepomnyaschikh. Schwartz alternating method for solving the singular Neumann problem [translation of computational algorithms in problems of mathematical physics (Russian), 99-112, Akad. Nauk SSSR Sibirsk, Otdel., Vychisl. Tsentr, Novosibirsk, 1985]. Soviet J. Numer. Anal. Math. Modelling, 5(1):69-78, 1990. Soviet Journal of Numerical Analysis and Mathematical Modelling. MR 1124937 (92k:65182)

[16] Walter Rudin. Functional analysis. International Series in Pure and Applied Mathematics. McGraw-Hill, Inc., New York, second edition, 1991. MR 1157815 (92k:46001)

[17] L. Shen and J. Xu. On a Schur complement operator arisen from Navier-Stokes equations and its preconditioning, volume 202 of Advances in computational mathematics (Guangzhou, 1997) Lectures Notes in Pure and Appl. Math. Dekker, New York, 1999. MR:1661553 (99i:76129)

[18] Qian shun Chang and Wei wei Sun. On convergence of multigrid method for nonnegative definite systems. J. Comput. Math., 23(2):177-184, 2005. MR2118053 (2005i:65207)

[19] T. Strouboulis, I. Babuška, and K. Copps. The design and analysis of the generalized finite element method. Comput. Methods Appl. Mech. Engrg., 181(1-3):43-69, 2000. MR1734667 (2000h:74077)

[20] T. Strouboulis, K. Copps, and I. Babuška. The generalized finite element method. Comput. Methods Appl. Mech. Engrg., 190(32-33):4081-4193, 2001. MR1832655(2002h:65195)

[21] Theofanis Strouboulis, Lin Zhang, Delin Wang, and Ivo Babuška. A posteriori error estimation for generalized finite element methods. Comput. Methods Appl. Mech. Engrg., 195(912):852-879, 2006. MR2195292 (2006i:65180)

[22] Andrea Toselli and Olof Widlund. Domain decomposition methods-algorithms and theory, volume 34 of Springer Series in Computational Mathematics. Springer-Verlag, Berlin, 2005. MR2104179 (2005g:65006)

[23] U. Trottenberg, C. W. Oosterlee, and A. Schüller. Multigrid. With contributions by A. Brandt, P. Oswald and K. Stuben. Academic Press Inc., San Diego, CA, 2001. MR:1807961 (2002b:65002)

[24] J. Xu and L. Zikatanov. The method of subspace corrections and the method of alternating projections in Hilbert space. J. Amer. Math. Soc., 15(3):573-597, 2002. MR1896233 (2003f:65095)

[25] Jinchao Xu. Iterative methods by space decomposition and subspace correction. SIAM Review, 34:581-613, 1992. MR 1193013 (93k:65029)

[26] Jinchao Xu and Ludmil T. Zikatanov. On multigrid methods for generalized finite element methods. In Meshfree methods for partial differential equations (Bonn, 2001), volume 26 of Lect. Notes Comput. Sci. Eng., pages 401-418. Springer, Berlin, 2003. MR2004013

[27] Kôsaku Yosida. Functional analysis. Die Grundlehren der Mathematischen Wissenschaften, Band 123. Academic Press, Inc., New York, 1965. MR0180824 (31:5054)

[28] Harry Yserentant. Old and new convergence proofs for multigrid methods. In Acta numerica, 1993, Acta Numer., pages 285-326. Cambridge Univ. Press, Cambridge, 1993. MR:1224685 (94i:65128) 
Department of Mathematics, University of California los Angeles, Los Angeles, CALIFORNia 90089

Current address: Department of Mathematics, Rutgers, The State University of New Jersey, Hill Center, Piscataway, New Jersey 08854-8019

E-mail address: leeyoung@math.rutgers.edu

Laboratory of Pure and Applied Mathematics, School of Mathematical Sciences, Peking University, Beijing 100871, People's Republic of China

E-mail address: jwu@math.pku.edu.cn

Department of Mathematics, Pennsylvania State University, McAllister Bldg., University Park, Pennsylvania 16802-6401 -and- Laboratory of Pure and Applied Mathematics, School of Mathematical Sciences, Peking University, Beijing 100871, People's Republic of China

E-mail address: xu@math.psu.edu

$U R L:$ http://www.math.psu.edu/xu/

Department of Mathematics, Pennsylvania State University, McAllister Bldg., UniVERsity Park, PenNSylvania 16802-6401

E-mail address: 1tz@math.psu.edu

$U R L:$ http://www.math.psu.edu/ltz 